\title{
A IMPORTÂNCIA DA FORMAÇÃO DO PEDAGOGO PARA O TRABALHO COM AS INFLUÊNCIAS CULTURAIS AFRICANAS DA PLANÍCIE GOITACÁ
}

\author{
Shayane Ferreira dos Santos ${ }^{*}$, Luis Carlos Soares ${ }^{1}$, Samantha Elias da Silva ${ }^{2}$ \& Larissa da \\ Silva Gomes ${ }^{2}$
}

\section{RESUMO}

SANTOS, S. F.; SOARES, L. C.; SILVA, S. E.; GOMES, L. S. A Importância da Formação do Pedagogo para o Trabalho com as Influências Culturais Africanas da Planície Goitacá. Perspectivas Online: Humanas \& Sociais Aplicadas, v.9, n.26, p.52-61, 2019.

O Brasil é um país miscigenado e multicultural. Entre as culturas responsáveis pela composição da brasileira, encontra-se a cultura africana, que muito contribuiu com a construção da identidade cultural do nosso país. Muitos são os reflexos da cultura africana e de suas recriações em nosso Brasil. Estas influências são evidentes nas danças, no vocabulário e no modo de ser do povo de Campos dos Goytacazes (RJ). Assim, o objetivo principal desta pesquisa é identificar as influências das raízes africanas nas manifestações culturais de Campos dos Goytacazes, ressaltando a importância delas para a formação dos futuros pedagogos. Pretendeu-se realizar um trabalho inicial em toda a bibliografia que aborda o assunto e logo após um trabalho de campo bastante intenso, através da realização de visitas e acompanhamento às manifestações culturais que ocorrem em território campista e que se configuram com influências africanas. Quanto aos aspectos metodológicos, o trabalho caracteriza-se por ser exploratório e bibliográfico. Quanto à abordagem do problema, apresenta-se de natureza qualitativa, com estudo de campo. Quanto às técnicas de coletas de dados, destacaram-se a observação participante, o questionário semi-estruturado aplicado às alunas do primeiro período do Curso de Pedagogia e entrevistas aos campistas participantes destes movimentos culturais. Como resultados, percebeu-se que as alunas revelaram maior conhecimento e reconhecimento acerca das influências africanas em manifestações culturais do universo campista, levando-as a construir uma sentimento de pertencimento e identificação com sua cidade. Além disso, este conhecimento impacta suas práticas profissionais, haja vista que são multiplicadoras em suas salas de aula.

Palavras-chave: Pedagogia; Cultura Africana; Formação do professor. 


\begin{abstract}
Brazil is a mixed and multicultural country. Among the cultures responsible for the composition of the Brazilian is the African culture, which contributed greatly to the construction of the cultural identity of our country. There are many reflections of African culture and its recreations in our Brazil. These influences are evident in the dances, vocabulary and way of being of the people of Campos dos Goytacazes (RJ). Thus, the main objective of this research is to identify the influences of African roots in the cultural manifestations of Campos dos Goytacazes, emphasizing their importance for the formation of future educators. It was intended to carry out an initial work in all the bibliography that addresses the subject and soon after a very intense field work, through visits and accompaniment to the cultural manifestations that occur in camper

territory and that are configured with African influences. As for the methodological aspects, the work is characterized by being exploratory and bibliographic. Regarding the approach to the problem, it is qualitative, with field study. Regarding data collection techniques, the participant observation, the semi-structured questionnaire applied to the students of the first period of the Pedagogy Course and interviews with the campers participating in these cultural movements stood out. As a result, it was noticed that the students revealed greater knowledge and recognition about African influences in cultural manifestations of the camper universe, leading them to build a sense of belonging and identification with their city. In addition, this knowledge impacts their professional practices, as they are multipliers in their classrooms.
\end{abstract}

Keywords: Pedagogy; African culture; Teacher training.

\footnotetext{
${ }^{1}$ Institutos Superiores de Ensino do CENSA - ISECENSA - Laboratório de Formação de Professor - LAFORP - Rua Salvador Correa, 139, Centro, Campos dos Goytacazes, RJ, CEP: 28035-310, Brasil.

${ }^{2}$ Alunas do Programa Voluntário de Iniciação Científica-PROVIC- Institutos Superiores de Ensino do CENSA - ISECENSA - Rua Salvador Correa, 139, Centro, Campos dos Goytacazes, RJ, CEP: 28035-310, Brasil.

(*) e-mail: shayaneferreira@yahoo.com.br

Data de recebimento:08/11/2019. Aceito para publicação: 15/11/2019.
}

Persp. Online: hum \& sociais aplicada., Campos dos Goytacazes, 26 (9) 52-61- 2019 


\section{INTRODUÇÃO}

Hoje, a educação abraça muitas causas e responsabilidades. Quando pensamos em todo o processo de construção da história brasileira, vemos o quanto a miscigenação está presente. Entre os povos participantes desta construção histórica e cultural, destaca-se o povo africano, negro, que veio com o objetivo de ser mão-de-obra escrava em terras brasileiras. Durante o tempo em que viveram aqui, muito contribuíram com a construção da identidade cultural do nosso país (SCHWARCZ, 2013).

Sabe-se que existem inúmeros reflexos da cultura africana e de suas recriações em nosso mundo. Quando olhamos para o Brasil, estas influências são evidentes em nossas danças, em nosso vocabulário, em nosso modo de ser, em nossa cultura (DEBUS, 2018)

Cultura congrega conhecimentos, artes, moral, leis, costumes, aptidões, hábitos adquiridos, herança cultural, tradição social, toda e qualquer necessidade básica como resposta ao ambiente, expressa modo de vida, povo, ocupação, territorialidade, instituições, linguagem, instrumentos, serviços e sentimentos (FIGUEIREDO, 2009).

Desta forma, muitos são os elementos que formam uma cultura e a valorização dela passa pelo conhecimento, passa pelo entendimento de como ocorreu a sua construção. No Brasil, há tempos, existe uma preocupação com esta temática e no ano de 2003 foi promulgada uma lei, a 10.639/03, alterada pela Lei 11.645/08, que tornou obrigatório o ensino da história e cultura afro-brasileira e africana em todas as escolas, públicas e particulares, do ensino fundamental até o ensino médio. Desta forma, o tema seria incorporado ao currículo escolar, sendo acessível a todos a possibilidade de compreensão acerca desta composição cultura.

Com esta lei, foi estabelecido um marco legal, político e pedagógico de reconhecimento e valorização das influências africanas na formação da sociedade brasileira e do protagonismo da população afro-brasileira na formação social, política e econômica do país (UNESCO, 2014).

A análise de nosso entorno, nossa cidade, revela esta presença forte da cultura negra e afrodescendente. Assim, revela-se com objetivo deste trabalho identificar as influências das raízes africanas nas manifestações culturais de Campos dos Goytacazes e sua importância na formação dos futuros pedagogas.

Para atender a este objetivo geral, traça-se os seguintes objetivos específicos: investigar, historicamente, as manifestações culturais existentes em Campos dos Goytacazes que apresentam raízes africanas; identificar o conhecimento das alunas do Curso de Pedagogia acerca das influências africanas na cultura de Campos dos Goytacazes; verificar a importância do estudo das influências africanas na construção da identidade campista, sob a percepção dos alunos.

Para isto, faz-se necessário partir do local para o global. Com as ações idealizadas, as alunas participantes do projeto conheceram as manifestações culturais campistas que são originárias da cultura afrodescendente. Desta forma, poderão ser multiplicadoras no Curso de Pedagogia do ISECENSA, no qual são alunas. Além disso, as alunas reconheceram suas origens, construindo um sentimento de pertencimento e identidade.

Persp. Online: hum \& sociais aplicada., Campos dos Goytacazes, 26 (9) 52-61- 2019 
Diante disto, o presente trabalho levantou a seguinte questão de pesquisa: de que forma o estudo das manifestações culturais campistas influenciadas pelos povos africanos contribui na formação dos futuros pedagogos?

As motivações deste estudo residiram no fato dos pesquisadores principais serem professores do Curso de Pedagogia, nas disciplinas de Artes e Psicologia da Educação, e entenderem a necessidade dos pedagogos - futuros educadores - terem este conhecimento de forma pessoal e profissional. Assim, têm conhecimento de suas origens, de suas influências culturais, construindo o sentimento de pertença a sua cidade e, consequentemente, sua cultura.

O impacto da cultura do povo português, com a cultura indígena, no caso de Campos dos Goytacazes, a nação dos Índios Goitacás, começa um processo de transformação, pois os cultos e danças africanas eram proibidas em solo brasileiro. Consequentemente os ritos de Candomblé africano passam a ser sincretizados, daí surgem os rituais de Umbanda, uma mistura ritualística do índio branco e o negro (SCHUWARCZ, 1993).

Encontra-se em Campos dos Goytacazes, na "Cidade de Palha", atual bairro de Custodópolis, um terreiro de Jongo e uma escola de samba prova viva dessa eugenia cultural. Além dessas manifestações oriundas da África, temos ainda a Mana Chica da região do CABOIO e o Folguedo do Boi Samba, único em todo o Brasil (FERRO, 2018).

Desta forma, Campos dos Goytacazes é um celeiro de influências culturais que precisam ser estudadas e multiplicadas, principalmente, entre as futuras professoras estudantes do curso de Pedagogia, pois elas serão multiplicadoras em suas salas de aula.

\section{METODOLOGIA}

A pesquisa caracterizou-se por ser exploratória, pois tem como objetivo oportunizar maior proximidade com o tema e problema (GIL, 1995). Além disso, configura-se como descritiva, pois busca descrever características de uma população ou fenômeno.

Quanto ao delineamento, revelou-se uma pesquisa bibliográfica, com levantamento de aportes teóricos que fundamentem o assunto discutido. Segundo Lakatos e Marconi (2010, p. 166), "a pesquisa bibliográfica não é mera repetição do que já foi dito ou escrito sobre certo assunto, mas propicia o exame de um tema sob novo enfoque ou abordagem, chegando a conclusões inovadoras".

Quanto à abordagem do problema, foi de natureza qualitativa, pois não requereu o uso de métodos e técnicas estatísticas. O ambiente natural foi a fonte direta para coleta de dados e o pesquisador foi o instrumento- chave (GIL, 1995).

Além disso, foi realizado um vasto estudo de campo, para reconhecimento das manifestações culturais em solo campista. Algumas inserções foram em campo, propriamente dito, com o apoio e responsabilidade dos professores colaboradores em levar e guiar a observação. Em outros momentos, as manifestações culturais foram levadas à faculdade como apresentação para as alunas do Curso de Pedagogia e os envolvidos foram entrevistados neste momento. Faremos uma descrição mais detalhada sobre as ações.

As alunas iniciaram a pesquisa fazendo levantamento de bibliografia a respeito do tema na biblioteca da instituição. Com a orientação dos professores, em reunião, discutiu-se a bibliografia do projeto. As alunas envolvidas realizaram um estudo aprofundado e registraram suas descobertas.

Persp. Online: hum \& sociais aplicada., Campos dos Goytacazes, 26 (9) 52-61- 2019 


\section{PERSPECTIVASonline}

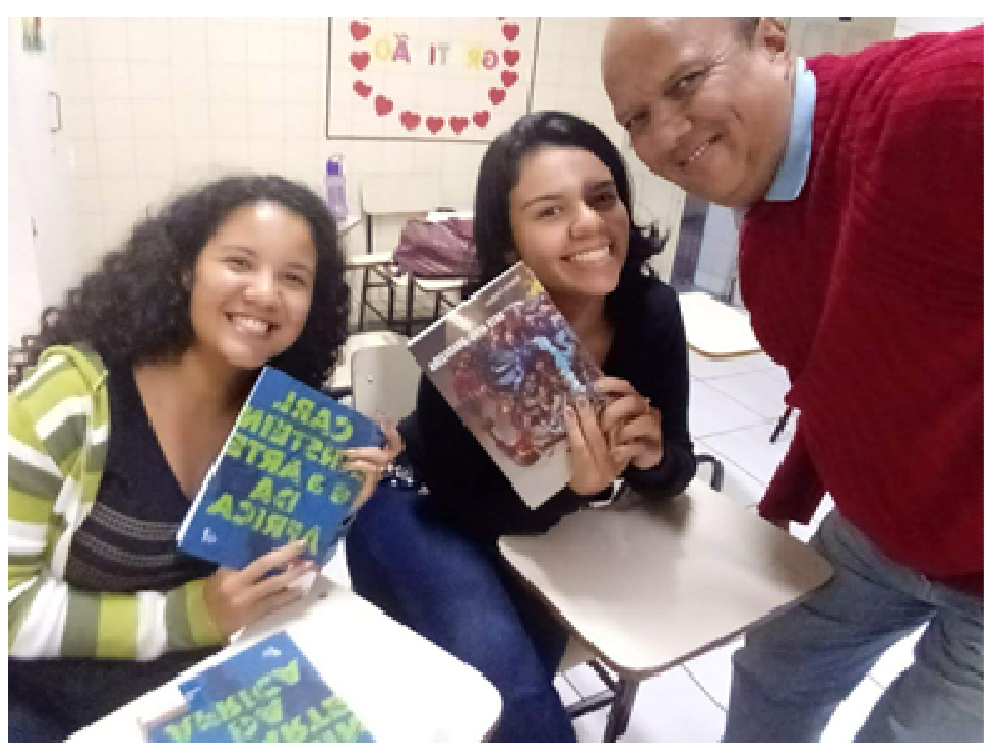

Figura 1: Registro do primeiro encontro: orientação de bibliografia

Em momento posterior, as alunas levaram estas descobertas para a sala de aula, na qual estão inseridas e, sob orientação do professor/orientador da pesquisa, as alunas realizaram um trabalho em forma de rotação por estações para aprofundamento de diferentes pontos dentro do tema central.

A prática de Rotação por Estações é definida como a organização dos alunos em grupos e cada um realiza uma tarefa, de acordo com o objetivo da aula proposto pelo professor. Estas atividades podem ser escritas, em forma de leituras, mas essencialmente um grupo, deverá estar envolvido com uma proposta on-line. Nesta atividade, é importante que o professor organize bem, pois a diversificação de materiais e propostas facilita a personalização do ensino. Após um determinado tempo combinado com os estudantes, eles devem trocar de "Estação". Este trabalho acontece até que todos os grupos passem por todas as estações (BACICH; TANZI NETO; TREVISANI, 2015).

$\mathrm{Na}$ etapa seguinte, as alunas realizaram uma pesquisa de campo na "Comunidade Teixeirão", reduto das manifestações culturais de Jongo e Mana Chica. Nesta visita, as alunas tiveram a oportunidade de conversar com dois profissionais fundamentais na manutenção e disseminação destas manifestações culturais. Estes são educadores patrimoniais e jongueiras, pertencentes a família Da Hora, que fundou há mais de trinta anos o grupo folclórico Ilê, que leva as raízes africanas nas suas apresentações.

Após este momento de investigação da realidade com as alunas envolvidas no projeto, a turma na qual elas estão inseridas realizou um um estudo sistemático sobre estas manifestações, proposto pela disciplina de Artes. Após o estudo, foi realizado um momento de discussões e debates sobre a relevância de inserir este estudo no currículo nas escolas da cidade.
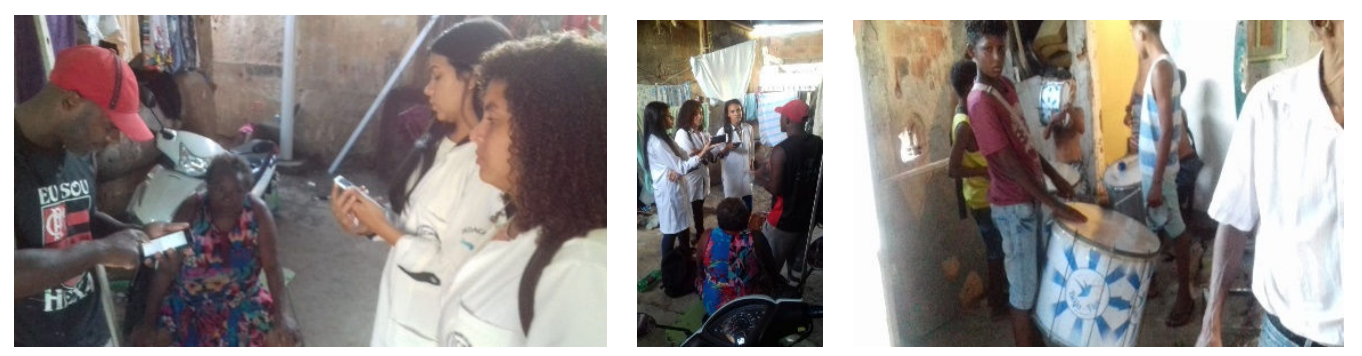

Persp. Online: hum \& sociais aplicada., Campos dos Goytacazes, 26 (9) 52-61- 2019 seer.perspectivasonline.com.br 


\section{PERSPECTIVAS Online CIENNIAS HUMANAS ESOCIAIS APLICADAS}

Figura 2: Registro das Visitas de Campo

Em seguida, as alunas foram à campo para pesquisar sobre as manifestações carnavalescas, destacando os "Bois Pintadinhos". Nesta oportunidade, visitaram a comunidade do Morrinho e conversaram com os líderes da comunidade sobre a mobilização das pessoas para a participação do "Boi Pintadinho" em todo carnaval. Nesta visita, puderam participar da construção de adereços para os Folguedos, entendendo este movimento cultural. Além disso, as alunas foram convidadas para participar dos ensaios da comunidade e retornaram num outro momento.

Como última etapa do estudo, as alunas tiveram a oportunidade de ler e discutir sobre a lei 10.639/03, alterada pela Lei $11.645 / 08$, que tornou obrigatório o ensino da história e cultura afro-brasileira e africana em todas as escolas, públicas e particulares, do ensino fundamental até o ensino médio. Assim, as alunas discutiram sobre a relevância do trabalho, que levou-as a conhecer as manifestações artístico-culturais de sua cidade, construindo conhecimentos para sua futura atuação profissional.

Para a conclusão desta pesquisa, as alunas envolvidas no projeto, junto com os professores e a turma na qual elas estão inseridas, organizaram um momento de culminância. Neste momento, será apresentado um teatro sobre o tema, com representação das manifestações culturais estudadas. Os profissionais entrevistados ao longo da pesquisa foram convidados para a culminância do trabalho.

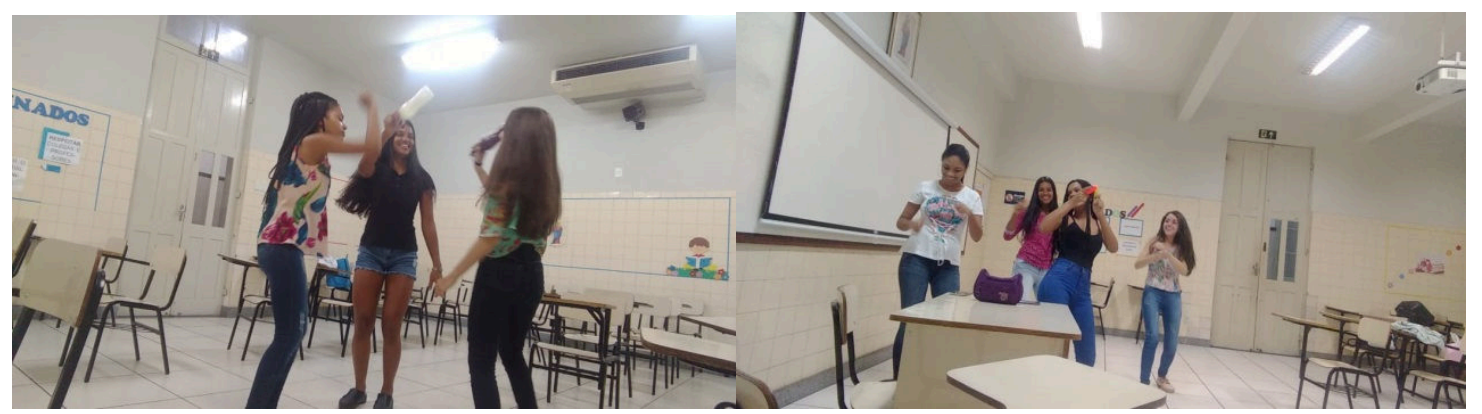

Figura 3: Ensaios para a Culminância do projeto

Quanto às técnicas de coletas de dados ao longo de todo o projeto, destaca-se a observação participante, pois o pesquisador estava inserido nos movimentos culturais. Além de questionário semi-estruturado aplicados às alunas do Curso de Pedagogia envolvidas diretamente com as ações do projeto, além da realização das entrevistas aos campistas participantes destes movimentos culturais.

\section{RESULTADOS E DISCUSSÕES}

Ao longo do trabalho, foi perceptível o crescimento das alunas no conhecimento das manifestações culturais de raízes africanas, além da valorização e entendimento de suas origens, desenvolvendo o sentimento de pertencimento.

Com relação a caracterização do público impactado pelo projeto, alunas no primeiro período de Pedagogia, nota-se que a maioria está inserida em um ambiente escolar, exercendo diferentes funções.

Persp. Online: hum \& sociais aplicada., Campos dos Goytacazes, 26 (9) 52-61- 2019 


\section{PERSPECTIVASonline}

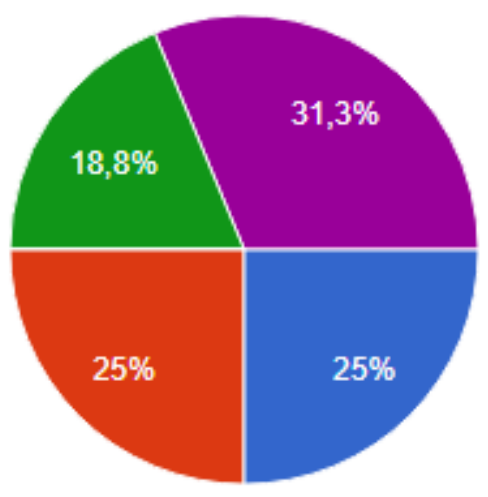

Não.

Sim. Sou professora de Educação Infantil ou Ensino Fundamental.

Sim. Sou mediadora de aluno com NEE.

Sim. Sou auxiliar de turma.

Sim. Exerço outra função na escola.

Grafico 1: Você atua na área pedagógica, em contato direto com os alunos?

Este dado corrobora a relevância da pesquisa realizada, pois são profissionais já em atuação e que não revelavam conhecimento a respeito dos assuntos abordados, conforme podemos constatar na próxima pergunta. Para mudança de tal realidade, é preciso investir na formação do professorado, para que a lei $10.639 / 03$, alterada pela Lei $11.645 / 08$, que tornou obrigatório o ensino da história e cultura afro-brasileira e africana, não fique apenas no pape

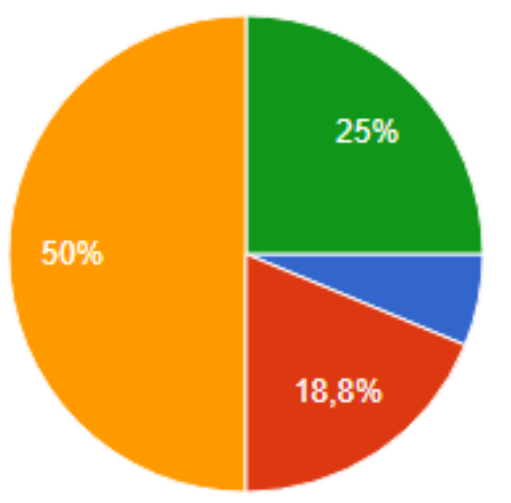

Sempre

Na maioria das vezes

Poucas Vezes

Nunca

Gráfico 2: Ao longo dos seus estudos, teve a oportunidade de estudar sobre as manifestações culturais de origem africana?

É perceptível a necessidade de se trabalhar com esta temática nos cursos de Formação de Professores, especialmente na formação inicial. Segundo Candau (2011), a formação pelo exemplo é a mais significativa no âmbito educacional. Em pesquisas realizadas pela autora, nota-se que a prática dos professores formadores contribui significativamente para a construção da identidade profissional dos licenciandos. Em sintonia com as ideias de Candau (2011), Rego e Mello (2002) afirmam que a formação do educador acontece numa simetria invertida, pois o professor aprende sua profissão, mas como aluno. Desta forma, práticas como as realizadas neste projeto são fundamentais.

Sobre as manifestações culturais de origem africana que as alunas passaram a conhecer com este projeto, detectou-se que, praticamente os alunos não as conheciam. As alunas destacaram como aprendizado o estudo do Maculelê, Jongo, Mana Chica, Boi Pintadinho, Capoeira, Bumba-Meu-Boi, Carreiros, Escolas de Samba, Folia de Reis. Além disso, acrescentaram as variedades das danças africanas, riqueza do vocabulário, a extensa

Persp. Online: hum \& sociais aplicada., Campos dos Goytacazes, 26 (9) 52-61- 2019 
cultura do continente em uma visão interna e externa ao interferir em outros países.

Ao serem questionadas se o estudo das influências culturais de Campos dos Goytcazes, RJ contribuiu para o futuro trabalho de pedagoga, destacam-se algumas respostas dadas:

\begin{abstract}
Aluna 1: "Sim, pois foi uma das etnias responsáveis pela formação do nosso país e merece respeito como todas as outras. Já que ultimamente vem enfrentando uma batalha para mostrar sua importância, a cultura africana merece ser repassada a todas, principalmente para as pedagogas e professoras que trabalharão ou até mesmo já trabalham com o futuro do nosso país".
\end{abstract}

Aluna 2: "Sim. Foi possivel adquirir um vasto conhecimento sobre as influências de tantas manifestações culturais no Brasil e na cidade de Campos dos Goytacazes. Dessa forma, enquanto futura pedagoga, poderei contribuir na formação de alunos que conheçam a cultura e as origens".

Aluna 3: "Sim, pois partindo de uma visão empirista, conhecemos o objeto de estudo a medida que temos experiência com o mesmo, isto é, para uma melhor compreensão das diversas culturas que temos contato em sala de aula, faz-se necessário a aprofundação de tais temas".

Aluna 4: "Sim, porque para transmitir o conhecimento eu preciso ser detentora dele, logo, contribuiu sim para passar este conhecimento para os meus alunos e explicá-los sobre as influências que a cultura africana tem sobre a nossa vida em Campos dos Goytacazes".

Quando de analisam as respostas obtidas, percebeu-se a importância que as alunas deram em ter este conhecimento e poderem construí-lo futuramente com seus alunos. Além disso, também destacaram o valor de ser um conhecimento construído na prática, a partir da inserção delas no campo de pesquisa. Este conhecimento empírico faz toda a diferença para a aprendizagem significativa.

É perceptível que os objetivos propostos para este trabalho foram alcançados de forma satisfatória, pois a cada etapa foi nítido o envolvimento das alunas e as descobertas que obtinham a cada visita, a cada entrevista. Os debates promovidos em sala de aula foram riquíssimos, fruto de estudo teórico-prático, levando às alunas ao empoderamento deste conhecimento.

Além disso, como resultado significativo, destaca-se a afirmação da grande maioria das alunas, em realizar práticas criativas com utilização de diferentes recursos pedagógicodidático para trabalharem futuramente, o tema desenvolvido neste trabalho. Ao longo dos encontros, narravam sobre a utilização de vídeos, filmes, cenas de novela como recurso de reflexão ao trabalho com a diversidade étnico-cultural. Conclui-se que, de fato, o projeto gerou impactos e rendeu os frutos esperados.

\title{
4. CONCLUSÃO
}

A construção do sentimento de pertencimento a uma cultura perpassa pelo conhecimento. A influência da cultura africana é diária em nosso vocabulário, danças, trejeitos, e muitas vezes, é esquecida ou marginalizada.

Levar futuras pedagogas ao encontro de suas raízes, ao (re)conhecimento de suas

Persp. Online: hum \& sociais aplicada., Campos dos Goytacazes, 26 (9) 52-61- 2019

seer.perspectivasonline.com.br 
origens é uma experiência que precisa ser vivida e repetida. Com base nos dados obtidos neste trabalho, a disciplina de Arte-Educação no Curso de Pedagogia do Isecensa sofrerá modificações em sua ementa, possibilitando o estudo teórico-prático destas influências da cultura africana nos aspectos culturais campistas.

Com relação ao objetivo específico investigar, historicamente, as manifestações culturais existentes em Campos dos Goytacazes que apresentam raízes africanas, foi possível constatar que a Mana Chica, o jongo, a capoeira, o samba são manifestações que foram construídas por meio destas influencias.

Sobre identificar o conhecimento das alunas do Curso de Pedagogia acerca das influências africanas na cultura de Campos dos Goytacazes, foi perceptível que era mínimo. Nota-se que este conhecimento era em nível do senso comum e por meio do projeto este conhecimento foi ampliado.

Quanto ao objetivo, verificar a importância do estudo das influências africanas na construção da identidade campista, sob a percepção dos alunos, percebe-se que foi unanimidade a relevância do trabalho. Todos os alunos destacam, em diferentes tons, que estudar estas influências o tornaram profissionais melhores, uma vez que se conhece suas origens, suas raízes e compreeende-se o porquê de muitas coisas.

Com relação à questão de pesquisa, responde-se que o estudo das manifestações culturais afro-descendentes enriquece grandemente o profissional de educação, pois além de criar identificação, permite o trabalho em combate ao preconceito, valorização das diferenças e individualidades.

Assim, mais uma vez, ressalta-se a necessidade de abordar esta temática na formação de professores. A medida que se conhece uma uma cultura, agimos contra a construção de pré-conceitos, favorecendo o respeito à diversidade. Percebe-se que o trabalho de estudo e conscientização realizado por esta pesquisa tende a surtir mais efeito, pois parte do conhecimento e do empoderamento dos sujeitos.

\section{REFERÊNCIAS}

BACICH, L.; TANZI NETO, A.; TREVISANI, F. Ensino híbrido: personalização e tecnologia na educação. Porto Alegre: Penso Editora, 2015.

BRASIL. Lei 10.639 de 09 de janeiro de 2003. Inclui a obrigatoriedade da temática "História e Cultura Afro-Brasileira e Africana" no currículo oficial da rede de ensino. Diário Oficial da União. Brasília, 2003.

CANDAU, V. M. A didática em questão. Petrópolis, RJ: Editora Vozes Limitada, 2011.

DEBUS, Eliane. A temática da cultura africana e afro-brasileira na literatura para crianças e jovens. Cortez Editora, 2018.

FIGUEIREDO, L. (Org.). Raízes africanas. Rio de Janeiro: Sabin, 2009 (Coleção Revista de História no Bolso).

FERRO, Neilda da Cunha Alves; DE OLIVEIRA, Thiago Soares. Colonização, cultura e língua em Campos dos Goytacazes. EntreLetras, v. 9, n. 3, p. 237-260, 2018.

Persp. Online: hum \& sociais aplicada., Campos dos Goytacazes, 26 (9) 52-61- 2019

seer.perspectivasonline.com.br 
GIL, A. C. Métodos e técnicas de pesquisa social. São Paulo: Atlas, 1995.

LAKATOS, E. M.; MARCONI, M. A. Fundamentos de metodologia científica. 7. ed. São Paulo. Ed. Atlas, 2010.

REGO, T. C.; MELLO, G. N. Formação de Professores na América Latina e Caribe: a busca por inovação e eficiência. In: CONFERÊNCIA INTERNACIONAL: DESEMPENHO DOS PROFESSORES NA AMERICA LATINA, TEMPO DE NOVAS PRIORIDADES. Anais... Brasília (DF), Julho, 2002. Disponível em: http://www.namodemello.com.br/pdf/escritos /oficio/teresaversaoenviada.pdf. Acesso em: 08 abr. 2018.

SCHWARCZ, Lilia Moritz. Nem preto nem branco, muito pelo contrário: cor e raça na sociabilidade brasileira. Editora Companhia das Letras, 2013.UNESCO.

Persp. Online: hum \& sociais aplicada., Campos dos Goytacazes, 26 (9) 52-61- 2019 seer.perspectivasonline.com.br 